\title{
Análise de Cluster
}

\section{Resumo}

Este trabalho procura resgatar procedimentos estatísticos multivariados aplicados aos processos de qualificação, especificação, avaliação, sistematização e instrumentalização de dados. Procuramos também analisar criticamente aspectos estatísticos, verificando como eles podem ser utilizados na avaliação, classificação e formação de conglomerados. Não tivemos a pretensão de esgotar o assunto, mas, tão somente, mostrar as perspectivas de um caminho que se descortina muito amplo, bastante complexo e pleno de significação. $\mathrm{O}$ trabalho apresenta um aspecto informal, que se evidencia pelos objetivos implícitos as suas normas intuitivas e julgamentos subjetivos e um aspecto formal, decorrente de objetivos bem formulados, presentes em comparações controladas por instrumentos matemáticos e estatísticos fidedignos. A contribuição do presente trabalho está em descrever, exemplificar e discutir a aplicação de várias técnicas hierárquicas aglomerativas, divisivas e particionais.

\section{Abstract}

This article tries to rescue multivariate statistical procedures applied to qualification, specification, evaluation systematization and instrumentation data process. We search also analyzing critically statistical aspects, by verifying as they can be utilized in the evaluation, classification, and formation of conglomerates. We didn't have the pretension of exhausting all the content, but just showing the way perspectives which is large complex and full of signification. This text presents on an informal aspect evidenced by the implicit objectives into the intuitive rules and subjective judgements on the other hand, is presented a formal aspect derived from well formulated objectives, present in controlled comparisons by valuable mathematics and statistical instrument. The contribution for the present work is describing, exemplifying and discussing an application of various agglomerative, divisible, hierarchical techniques.

\section{Definição de Cluster}

Não há, de fato, nenhum consenso universal do que constitui um cluster. O significado de tais definições como cluster ou semelhança depende de julgamentos de valores, em última instância, pelo pesquisador ou profissional. Nesta discussão de análise de cluster, adotaremos uma definição intuitiva.

Para ilustrar, considere os objetos como pontos em um espaço $\mathbf{p}$ dimensional, com cada uma das $\mathbf{p}$ variáveis, representado por um dos eixos do espaço. Um sistema de p coordenadas dimensionais é definido agora no espaço pelos valores das variáveis por objeto. Podemos, então, definir clusters como regiões contínuas que aparecem na massa relativamente grande de pontos no espaço, isto é, regiões com alta densidade de pontos, separadas de outras por regiões com pequena massa relativa (baixa densidade de pontos). O termo "cluster natural" freqüentemente descreve agrupamentos fundados neste tipo de argumento. (Wang, 1978)

Para definir "clusters naturais", não impomos nenhuma restrição, a priori, na estrutura dos dados. Por conseguinte, aos dados é permitido ditar os padrões de agrupamento. Isto é contrastante com outras definições propostas que afirmam que só poderiam ser chamados de cluster objetos alocados por proximidade, isto é, objetos em um cluster deveriam estar mais próximos uns

\footnotetext{
* Mestre em Educação e Tecnologia - Unicamp. Bacharel e Licenciado em Física. Professor dos cursos de Administração, Ciências Contábeis e Comércio Exterior das Faculdades Integradas "Campos Salles".
} 
aos outros que para objetos em outro cluster. Passa a ser restritiva, então, a sensação de que podemos ter dificuldade de identificar clusters diferentes na variedade esférica. A figura 1 mostra dois jogos de dados que exibem estruturas diferentes. Os presentes clusters são bastante óbvios de definir e a maioria das técnicas de agrupamento não teriam nenhum problema em descobri-los. Porém, procedimentos que enfatizam o critério de proximidade demorariam um certo tempo recuperando os clusters naturais mostrados na figura 1. (Ahlberg,1997)

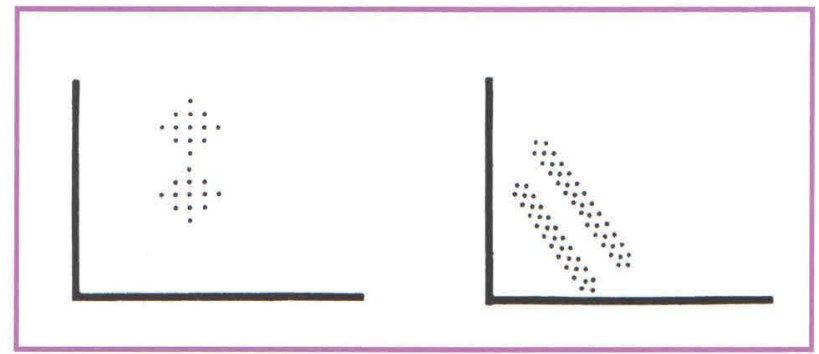

Figura 1

\section{Objetivos da Análise de Cluster}

A análise de cluster objetiva solucionar o seguinte problema: dada uma amostra de $\mathbf{n}$ objetos, cada um dos quais caracterizados por $\mathrm{p}$ variáveis; devemos criar um critério para se agrupar os objetos em classes, de forma que objetos que possuam características semelhantes estejam na mesma classe. $\bigcirc$ método deve ser quantitativo e o número de classes não é conhecido.

O objetivo básico da análise de cluster é encontrar os agrupamentos naturais de indivíduos (ou objetos, ou pontos, ou elementos químicos, ou espécies biológicas, ou unidades etc.). Um agrupamento de indivíduos devidamente caracterizados pode formar uma população completa ou pode ser uma amostra de alguma população maior. Mais formalmente, a ànálise de cluster objetiva alocar indivíduos em grupos de elementos mutuamente exclusivos, semelhantes, isto é, agrupa-se tal que os elementos pertencentes a um grupo são mais parecidos quanto possível uns com outros, enquanto indivíduos em grupos diferentes são dissimilares. Isto nos leva a medir a semelhança (ou diferença) de todo par de indivíduos. As seme- lhanças, às vezes, são observadas diretamente, enquanto em outros casos elas são derivadas de uma matriz de dados de um modo apropriado. (Chatfield, 1992)

Quando só duas variáveis estão consideradas em cada elemento, é relativamente fácil representar os dados e escolher os cluster através de processos puramente visuais. É intuitivo reunir em três grupos os dados bivariados representados no gráfi$\mathrm{co}$, conforme ilustra a figura 2 :

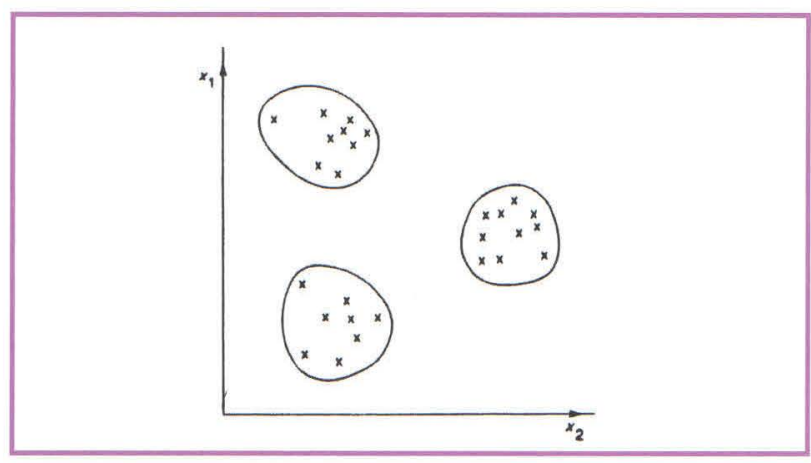

Figura 2

Podem ser subdivididos os grupos que formam uma "partição" em grupos menores ou maiores, de forma que, para uma eventual finalidade, cria-se uma estrutura hierárquica completa daquele determinado grupo de indivíduos. Esta estrutura é chamada freqüentemente de "árvore hierárquica" ou "dendograma", e pode ser representada num diagrama, como ilustra a figura 3 :

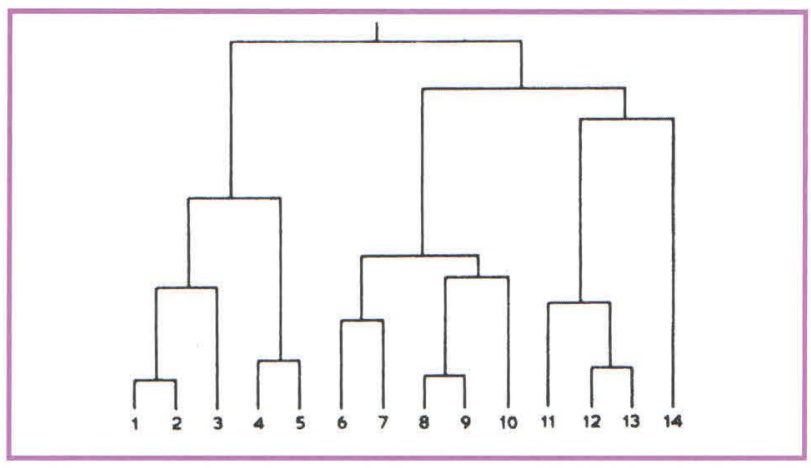

Figura 3

É também objetivo desta análise descobrir o inter-relacionamento que existe entre variáveis. Devem ser escolhidos elementos cujas medidas a serem consideradas sejam homogêneas. Não há 
nenhum interesse na possibilidade dos elementos se agruparem em características que exibirem diferenças sistemáticas. Em muitas colocações de aplicação, porém, há razão para acreditar que o jogo de elementos pode ser dividido em grupos e em subgrupos que diferem de modos significantes. Então, podemos definir que o termo comumente usado para o somatório de procedimentos que buscam separar os dados de componentes em grupos é a Análise de Cluster. (Asahi, 1994)

\section{Estágios da Análise de Cluster}

A figura 4 mostra os estágios básicos de uma análise de cluster. $\bigcirc$ processo começa levando as medidas das $\mathbf{p}$ variáveis em cada um dos $\mathbf{n}$ objetos. A matriz $\mathbf{n} \times \mathbf{p}$ de dados é transformada, então, em uma matriz $\mathbf{n} \times \mathbf{n}$ de semelhança ou, alternativamente, por medidas de distância onde são computadas as semelhanças ou distâncias entre pares de objetos pelas variáveis de p. Logo, um algoritmo é selecionado com a finalidade de definir as regras que concernem ao agrupamento dos objetos em subgrupos com base nas semelhanças. $\mathrm{O}$ objetivo, em muitas aplicações de cluster, é chegar a grupos de objetos que exibem variação pequena dentro do próprio cluster, relativa à variação entre os clusters. Como um passo final, os resultados dos clusters são contrastados em termos dos valores médios das p variáveis, ou de outras características de interesse.

Há dois problemas diretos na aplicação do procedimento descrito acima. Primeiro, precisamos definir uma medida de semelhança entre objetos, mas isto requer que a definição "semelhança" seja

\section{1e estágio}

\begin{tabular}{|c|c|c|c|c|c|c|}
\hline \multirow{6}{*}{ Objetos } & \multirow{5}{*}{$\begin{array}{l}\mathrm{O}_{1} \\
\mathrm{O}_{2} \\
\mathrm{O}_{3}\end{array}$} & \multicolumn{5}{|c|}{ Variáveis } \\
\hline & & $X_{1}$ & $X_{2}$ & $X_{3}$ & $\cdots$ & $X_{n}$ \\
\hline & & & & & & \\
\hline & & & & & & \\
\hline & & & & & & \\
\hline & $\mathrm{O}_{\mathrm{n}}$ & & & & & \\
\hline
\end{tabular}

\section{2- estágio}

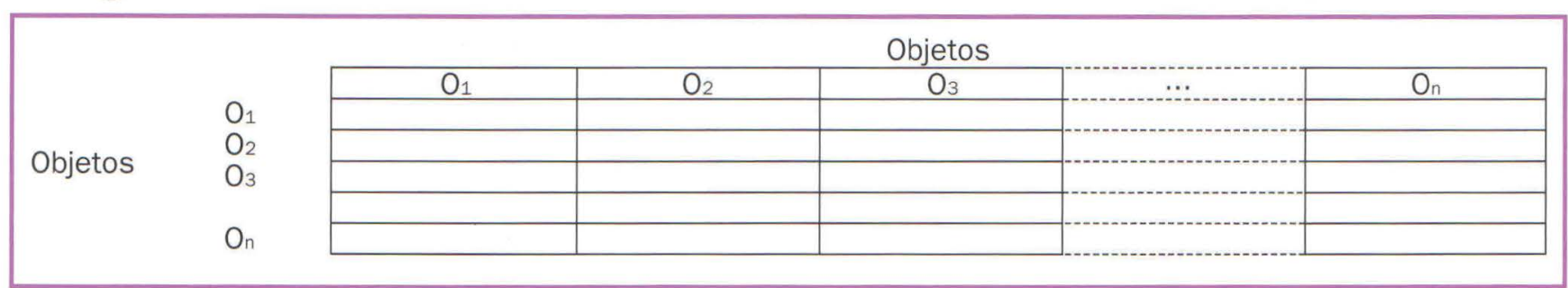

\section{3ㅇe estágio}

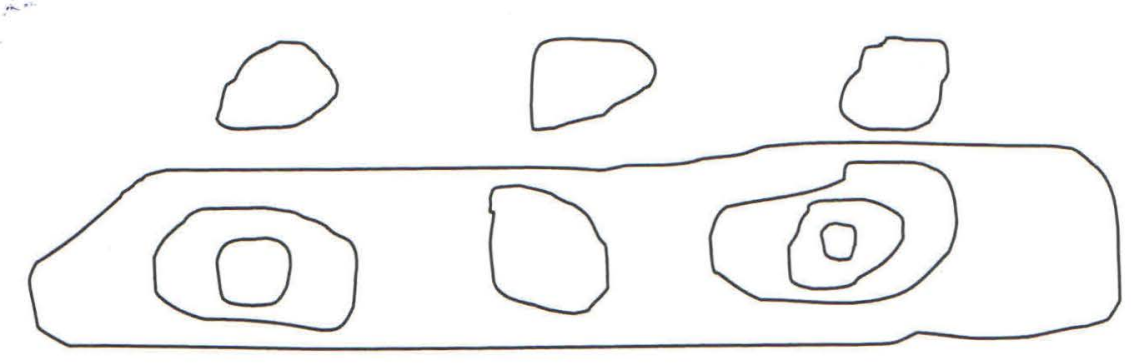




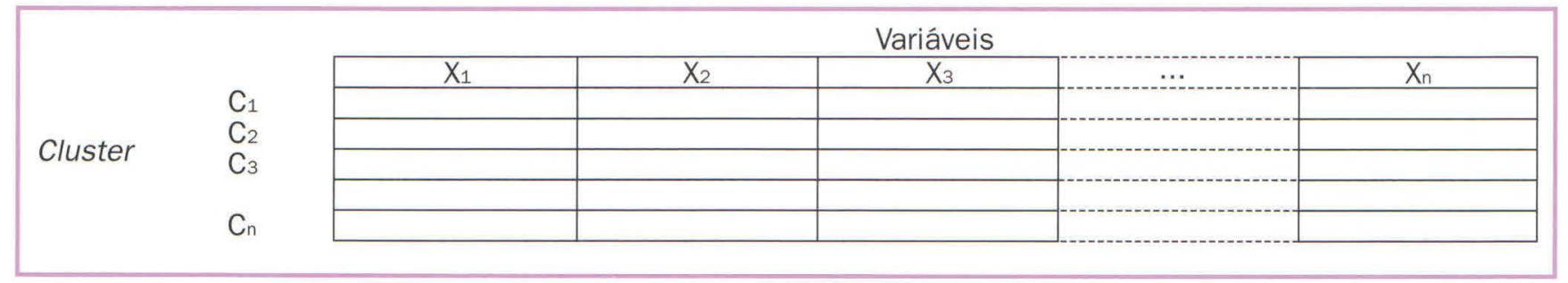

Figura 4 - estágio (1): $\mathbf{n}$ objetos mensurados com p variáveis; estágio (2): transformação dos dados numa matriz de distâncias $\mathbf{n} \times \mathbf{n}$; estágio (3): formação dos clusters; estágio (4): perfil dos clusters.

válida e significativa, o que não é comumente fácil. Um segundo ponto, é a especificação de um procedimento para formar os clusters baseado na medida escolhida de semelhança. Deveremos mostrar, porém, que há dúzias de medidas de semelhança que foram usadas em aplicações de agrupamentos e, literalmente, existe uma lista aparentemente infinita de possíveis algoritmos hierárquicos ou mutuamente exclusivos. Compreensivelmente, nossa discussão em certas instâncias será incompleta. Porém, discutindo alguns dos critérios de semelhança mais comuns, proveremos um tratamento representativo e informativo. (Evaritt, 1980)

\section{Finalidades da Análise de Cluster}

Foi sugerida uma variedade de usos para a análise de agrupamentos. Eles incluem: exploração de dados, redução de dados, geração de hipótese e predição baseada em grupos.

A primeira finalidade é auto-explicativa. A pessoa começa freqüentemente uma análise de cluster com uma pequena idéia clara de como um grupo de indivíduos é estruturado, de forma que o método pode ser visto como uma ferramenta de constatação e aprimoramento. Se os elementos de um cluster se mostram parecidos, então podemos reduzir a informação sobre os $\mathbf{n}$ indivíduos para mostrar um número muito menor de dados. Esperançosamente, a análise pode gerar algumas idéias (ou hipóteses) relativas à estrutura da população. Em particular, o número de cluster sugerido pela primeira análise pode ser tratado como uma hipótese a ser testada por um grupo novo de dados. Às vezes, podem ser usados os cluster para uma análise de predicativos mais recentes. Falando em termos gerais, podemos dizer que uma análise de cluster terá êxito, se trouxer informações para aprimorar agrupamentos previamente determinados em um grupo de dados, ou ajudar a formalizar sua estrutura hierárquica. (Abram, 1995)

\section{Medidas de semelhança}

Fundamental ao uso de qualquer técnica hierárquica é a computação de uma medida de semelhança ou dessemelhança entre os respectivos objetos. Estas medidas podem ser separadas em duas largas classes fundadas na qualidade dos dados disponíveis. Com dados que têm propriedades métricas, pode ser usada uma medida de "distância" e, se considerarmos dados que têm componentes qualitativos, uma medida de "comparação" é apropriada.

\section{Técnicas de agrupamentos}

Uma vez que um pesquisador concordou na escolha de uma medida de semelhança apropriada, o próximo passo é selecionar um tipo particular de algoritmo computacional. Um número bastante grande de algoritmos aglomerativos foi proposto. Maiores detalhes encontram-se nas revisões de Cormack (1971), e Hamacher (1993). Serão discutidos dois dos tipos comuns de técnicas de cluster. No primeiro grupo, as chamadas técnicas hierárquicas e, no segundo grupo, as técnicas chamadas particionais. As técnicas hierárquicas produzem um dendrograma como é mostrado na figura 3. Estas técnicas 
começam com o cálculo das distâncias de cada indivíduo a todos os outros indivíduos. Grupos são formados então por um processo de aglomeração ou divisão. No processo de aglomeração, todos os grupos começam com um só indivíduo. São unidos, então, gradualmente, indivíduos de mesmas características até que todos os indivíduos estejam em um único grupo. No processo divisivo, todos os indivíduos começam em um único grupo. Este grupo é então dividido em dois, os dois grupos resultantes são divididos em três ou quatro, e assim por diante até que todos os objetos formem seus próprios grupos. A segunda aproximação para se obter um cluster envolve partições: começamos definindo centros de grupos arbitrários, e alocamos os indivíduos no grupo mais próximo. São calculados então novos centros, e indivíduos são permutados a novos grupos, se estiverem mais próximos ao centro. $\mathrm{O}$ processo continua até que a estabilidade seja alcançada com um número predeterminado de grupos. Normalmente, a amplitude dos valores é experimentada, para se chegar a um número final de cluster.

\section{Aproximações visuais para achar um cluster}

Para exemplificar, consideraremos observações com dados bivariados, o mais simples e, provavelmente, o melhor modo de achar qualquer cluster natural. Traçamos o gráfico cartesiano e definimos os clusters visualmente. $\mathrm{O}$ juízo subjetivo pode ser usado para identificar qual- quer cluster presente. Às vezes, os clusters são visualmente óbvios, outras vezes, precisaríamos de um processo computacional para nos auxiliar. Isto é particularmente verdade, quando os clusters são encurvados ou se prolongam, como ilustra a figura 5 . $\bigcirc$ olho humano percebe mais facilmente clusters de forma esférica, mas não está tão apto a descobrir grupos de dados homogeneamente distribuídos onde não há nenhum cluster natural, ou, ainda, poderia descobrir clusters espúrios, fruto de uma aproximação, normalização ou regressão de algoritmos. Se a presença de um cluster é duvidosa, pode ser aconselhável levar um teste formal de hipótese, difundindo fortuitamente os pontos num plano, como no processo de Poisson. Muitas pessoas poderiam suspeitar que os pontos na figura 6 foram colocados ao acaso, mas de fato foram gerados por um modelo chamado de hard-core, onde não são permitidos pontos cuja distância mínima entre eles seja R. A figura 7 foi obtida com os dados de um processo de Poisson. (Robertson, 1991)

Com mais de duas variáveis, podem ser usadas as técnicas para adquirir uma representação bidimensional dos dados e, conseqüentemente, achar os clusters visualmente. Dos métodos diretos bidimensionais, o uso das curvas de Andrew parecem ser melhores para identificar cluster. Os métodos de ordenação indiretos também podem ser muito úteis. Se foram registrados dados de distância, podemos tentar usar uma escala clássica ou ordinal em duas dimensões, para traçar o gráfico e procurar os clusters. Se os dados foram registrados numa matriz ( $\mathbf{n} \times \mathbf{p})$, podemos tentar

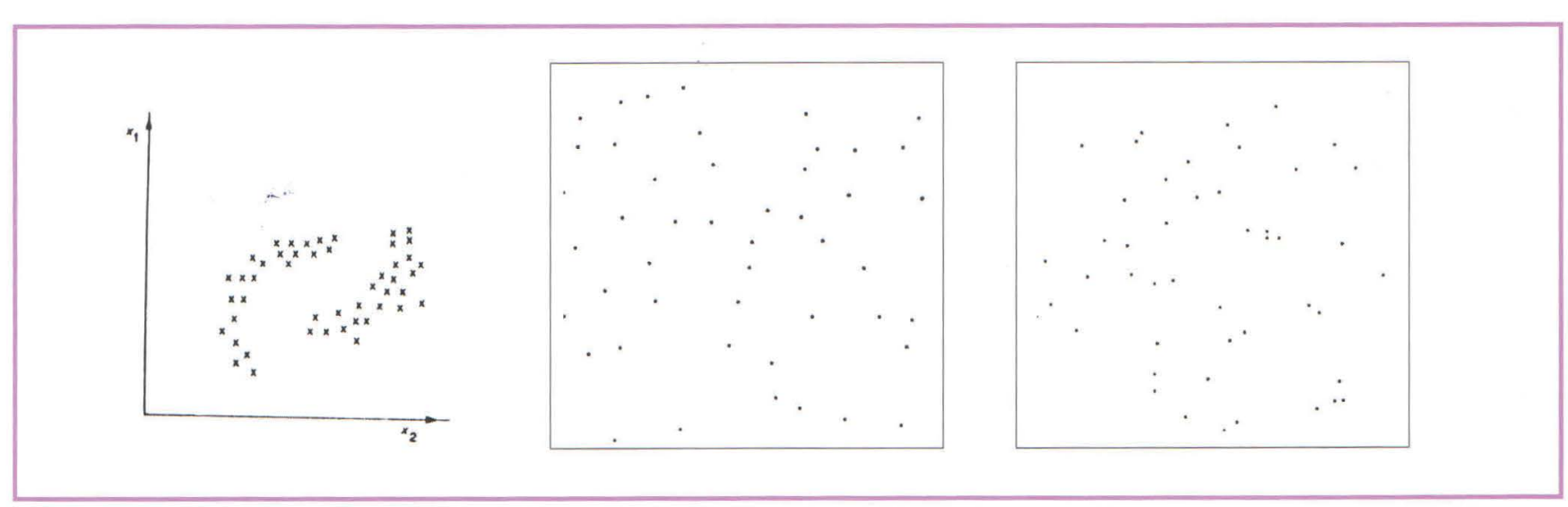


a Análise de Componente Principal. Se for concluído que os primeiros dois componentes (por exemplo) caracterizam o fato, e são responsáveis pela discrepância total, podemos conspirar que as pontuações destes dois componentes para cada indivíduo formem pares ordenados, permitindo obter um gráfico cartesiano que possibilitará procurar os clusters visualmente. Se mais de dois componentes forem necessários para explicar satisfatoriamente os dados, então a Análise de Componente Principal ainda será útil para tentar com mais segurança um algoritmo de aproximação nas pontuações dos primeiros componentes importantes, que representarão os dados originais.

Não são enfatizadas as técnicas de aproximações visuais na literatura, bem como nas pesquisas, talvez por causa do elemento subjetivo envolvido.

\section{Técnicas hierárquicas}

As técnicas hierárquicas começam com o cálculo das distâncias de cada indivíduo a todos os outros indivíduos. Grupos são formados então por um processo de aglomeração ou divisão.

\section{Métodos hierárquicos aglomera- tivos}

No processo hierárquico de aglomeração, todos os grupos começam com um só indivíduo. São unidos então, gradualmente, indivíduos de mesmas características, até que todos os indivíduos estejam em um único grupo.

\subsection{Método hierárquico aglomerativo - single-link}

O método hierárquico aglomerativo do single-link utiliza uma regra de mínima distância, que começa unindo os dois indivíduos mais próximos, que constituirão o primeiro agrupamento. $\mathrm{Na}$ próxima fase, duas coisas podem acontecer: ou um terceiro indivíduo se unirá ao agrupamento já formado, ou outros dois indivíduos que agora estão mais próximos se unirão para formar um segundo agrupamento. A decisão é ditada pelo critério da distância mínima entre os elementos do grupo. $\mathrm{O}$ processo repete-se até que todos os indivíduos pertençam a um só grupo.

\subsection{Método hierárquico aglomerativo - complete linkage ou furthest neighbor}

O método hierárquico aglomerativo - complete linkage - é precisamente oposto ao single-link na escolha da distância. A distância considerada agora é a maior entre um par de indivíduos.

\subsection{Método hierárquico aglomerativo - average linkage}

O método hierárquico aglomerativo - average linkage, também conhecido como weighted pair-group average - é outra variação dos algoritmos de single e complete linkage. Este algoritmo segue a mesma forma de união dos métodos descritos anteriormente, porém a distância entre agrupamentos é definida como a distância média entre os pares.

\subsection{Método hierárquico aglomerativo de ward ou método da soma dos quadrados dos erros}

O Prof. Ward, da Stanford University, propôs em 1963 um método para formar clusters baseado no resultado da perda de informações gerada pelo agrupamento de indivíduos, medido pela soma total das diferenças quadradas de todos elementos do agrupamento para o qual o indivíduo pertence. A regra para união está em aumentar a soma de erro dos quadrados, verificada na combinação de todo par possível de agrupamentos. Este valor é chamado de E.S.S. (error sun of square) e será usado como uma função objetiva.

O algoritmo também desenvolvido pelo Prof. Ward emprega um procedimento de agrupamentos hierárquicos. O processo de agrupamento começa considerando $\mathrm{k}$ grupos de um só elemento. O primeiro cluster é formado selecionando os 
dois destes $\mathbf{k}$ grupos que quando unidos produzam a menor depreciação no valor da função objetiva. Estes k - 1 grupos restantes são então reexaminados para determinar quais serão os próximos dois (desses $\mathrm{k}$ - 1 grupos) a serem unidos para minimizar o aumento na função objetiva. Os $\mathbf{k}$ grupos iniciais são assim reduzidos de $\mathbf{k}$ para $\mathbf{k}-1$; de k-1 para $\mathbf{k}-2$; e assim, sucessivamente, até que reste apenas um grupo 1 de $\mathrm{k}$ elementos. Em cada fase do procedimento (de $\mathbf{k}$ para $\mathbf{k}-1$ ), o valor da função objetiva é avaliado. Mudanças neste valor de estágio para estágio provêem um fator importante para determinação do número "natural" de clusters para os $\mathbf{k}$ elementos.

\section{Métodos hierárquicos divisivos}

Uma classe de métodos, chamada binary splitting ou métodos hierárquicos divisivos, normalmente opera por meio de um algoritmo divisivo, e é apropriada, quando a maioria das variáveis são de atributos binários. Embora algoritmos divisivos geralmente sejam menos eficientes que algoritmos de aglomerativos, em alguns casos são usados com bastante eficiência. Por exemplo, tentando classificar um grupo de adultos com deficiência física, por ser importante saber se eles são capazes de subir escadas ou não. Esta variável binária pode ser usada para dividir facilmente a amostra em dois grupos. Uma segunda variável binária, como a capacidade auditiva ou visual, pode ser usada para avançar na subdivisão de cada grupo em dois. Um exemplo claro é descrito por Jones(1979) que utiliza este procedimento com diversas variáveis binárias, objetivando escolher quais variáveis dividiriam a amostra em dois subconjuntos da melhor forma possível.

Métodos hierárquicos divisivos consideram inicialmente que tođos os indivíduos estejam alocados num único cluster, e começam dividindo todo grupo de indivíduos em dois grupos. Um problema imediato é como executar a primeira divisão. Com $\mathbf{n}$ indivíduos há $2^{\mathrm{n}-1}$ maneiras diferentes para formar subconjuntos de 2 elementos. Conseqüentemente, até mesmo com a utilização de computadores de última geração, o número de subconjuntos considerado terá que ser restrito na maioria dos problemas práticos que envolvem um número grande de variáveis. Uma vez que a divisão inicial é feita, são movidos elementos de um agrupamento para outro, ou são feitas melhores subdivisões dos agrupamentos já formados. O que distingue os vários métodos de divisivos é: (1) como a divisão inicial é efetuada, e (2) como formar subdivisões de agrupamentos.

\subsection{Método hierárquico divisivo - splinter-average distance}

Smith Mac Naughton, em 1962, propôs um método baseado em computar a distância média entre todos os indivíduos num pedaço do grupo, e a distância média destes mesmos indivíduos para os demais elementos do grupo. $O$ processo começa separando o indivíduo que tem a distância maior dos outros elementos. Assim, são formados dois grupos. Em seguida, são computadas as distâncias médias entre o indivíduo separado e os demais elementos do grupo principal, bem como as distâncias médias de cada indivíduo no grupo principal para os outros indivíduos do mesmo grupo; se a distância entre um indivíduo do grupo principal e o indivíduo do grupo separado for menor que a distância entre este indivíduo e os elementos de seu grupo, é razoável que ele deva ser removido e alocado neste grupo separado. Uma vez que a composição dos dois agrupamentos foi definida, isto é, a distância média de cada indivíduo para seu próprio agrupamento é menor que a distância média para indivíduos de outro agrupamento, o processo é reciclado em cada um dos cluster, até termos $\mathbf{n}$ clusters de um só elemento.

\subsection{Método hierárquico divisivo de interação automática (aid . automatic interaction detection)}

Morris Sonquist e Bernard Morgan, em 1964, dividiram uma amostra em subgrupos mutuamente exclusivos por uma série de divisões binárias. Cada divisão é determinada selecionando uma variável independente e suas categorias que 
maximizam a redução da variação inexplicada na variável dependente. $\mathrm{O}$ resultado, no fim deste processo, é ter um grupo com baixa pontuação de critério e outro com alta pontuação de critério.

O processo começa considerando, para cada variável independente, a melhor separação definida em termos de razão $\mathrm{B}_{\mathbf{c}} / \mathrm{T}_{\mathbf{c}}$, onde $\mathrm{B}_{\mathbf{c}}$ é a soma dos quadrados intergrupos, $\mathbf{T}_{\mathbf{C}}$ é a soma total dos quadrados (a subscrição c é usada para definir a variável de critério, também conhecida como variável dependente). Uma vez que as divisões binárias foram efetuadas para cada variável, a variável com a razão $\mathrm{B}_{\mathbf{C}} / \mathrm{T}_{\mathcal{C}}$ mais alta é usada para dividir a amostra, e todas as outras divisões são descartadas. Logo, cada subgrupo é tratado como uma amostra inicial separada, e o processo é repetido naquele subgrupo que possuir a soma dos quadrados totais maiores que a soma dos quadrados da variável de critério. $O$ processo termina, se o $\mathrm{B}_{\mathbf{c}}$ para aquele grupo for maior que o $\mathrm{T}_{\mathbf{c}}$.

\section{Técnicas particionais}

Ao contrário das técnicas hierárquicas, são métodos que efetuam uma partição dos dados sem requerer que a colocação de um indivíduo num cluster seja irreversível, quer dizer, podem ser realocados indivíduos, se a configuração inicial realmente for inexata. Estas técnicas particionais dividem um banco de dados otimizando algum critério formal predefinido. $O$ uso das técnicas particionais normalmente assumem que o número de agrupamentos finais seja conhecido e especificado no início, embora alguns métodos permitam que este número possa variar durante o curso da análise. As técnicas particionais diferem com relação a: (1) como os agrupamentos são iniciados; (2) como são alocados indivíduos e, (3) como alguns ou todos os indivíduos já alocados podem ser recolocados em outros clusters.

Foram propostos vários métodos para se iniciar os clusters, incluindo o Método dos Centróides. Se queremos que uma partição contenha g clusters, podemos escolher $\mathrm{g}$ pontos para definir os primeiros clusters que serão rearranjados futuramente.
Os pontos (ou indivíduos) restantes são alocados a um dos clusters iniciais, normalmente pela mínima distância euclidiana (se possível), ou então por critérios aleatórios ou pessoais. A recolocação por interatividade entre indivíduos continua até que algum critério seja satisfeito.

A figura 8 mostra que a variação entre clusters pode ser inferida avaliando a distância entre os centróides dos clusters e os objetos do espaço (a representação na figura 8 é somente em duas dimensões, enquanto os clusters são geralmente multidimensionais). Para entender como este critério pode ser usado para formar clusters, começaremos com um determinado número de centros de clusters, que pode ser escolhido arbitrariamente ou propositadamente, alocando objetos ao mais próximo centróide.

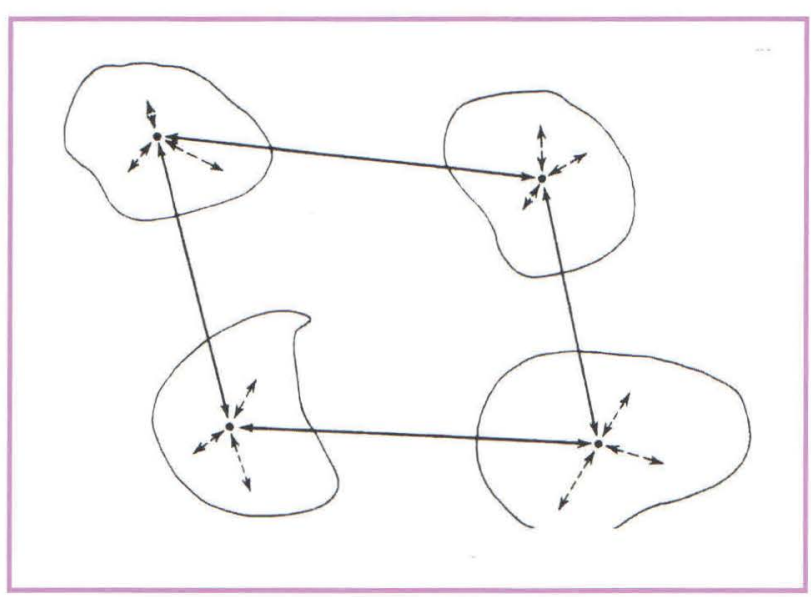

\section{Figura 8}

O método hierárquico particional conhecido como método " $k$ - means" e o método hierárquico particional baseado na "trilha" (ou "determinante") são os mais utilizados.

\section{Problemas de Análise de Cluster}

Há muitos algoritmos para análise de cluster. Porém, não há nenhum consenso do melhor método. Infelizmente, algoritmos diferentes necessariamente não produzem os mesmos resultados em um determinado jogo de dados. Há um grande componente subjetivo nos resultados de qualquer método em particular.

Um teste válido de qualquer algoritmo é levar 
um jogo de dados com uma estrutura de grupo conhecida, e verificar se o algoritmo pode reproduzir esta estrutura. Porém, este teste só trabalha com casos de classificação muito distintos. Quando há uma sobreposição considerável entre os elementos dos grupos iniciais, uma análise de cluster pode produzir uma solução que é bastante diferente da verdadeira situação.

Algumas dificuldades surgirão por causa da forma de cluster. Por exemplo, suponha que haja duas variáveis $\mathrm{X}_{1}$ e $\mathrm{X}_{2}$; traçando o respectivo gráfico cartesiano, poderemos ter alguns possíveis padrões como mostra a figura 9 . O caso (a) provavelmente será confirmado por qualquer algoritmo razoável, como também o caso (b). No caso de (c), alguns algoritmos não poderão definir dois clusters por causa dos pontos intermediários. Já nos casos (d), (e) e (f), a maioria dos algoritmos teria dificuldade em definir os clusters, e, provavelmente, apresen- tariam diferentes resultados. Se refletirmos um pouco mais, verificaremos que visualmente estes grupos de dados poderiam representar vários problemas práticos que, dependendo da natureza do problema, poderiam ser divididos de forma completamente diferente, atendendo a necessidades específicas, ou mesmo utilizando critérios puramente subjetivos.

Claro que a análise de clusters só pode estar baseada em variáveis que são determinadas por dados coletados. Então, elas devem ser pertinentes à classificação requerida. Classificar discentes definindo variáveis como altura, peso, ou capacidade pulmonar pode ser inútil, quando se tratar de aprendizado em línguas. Estas variáveis talvez sejam mais úteis para a cadeira de Educação Física. Porém elas podem detectar certos tipos de anomalias biológicas que poderão comprometer o aprendizado de línguas. É um problema polêmico que requer muitas pesquisas, porém o que estamos
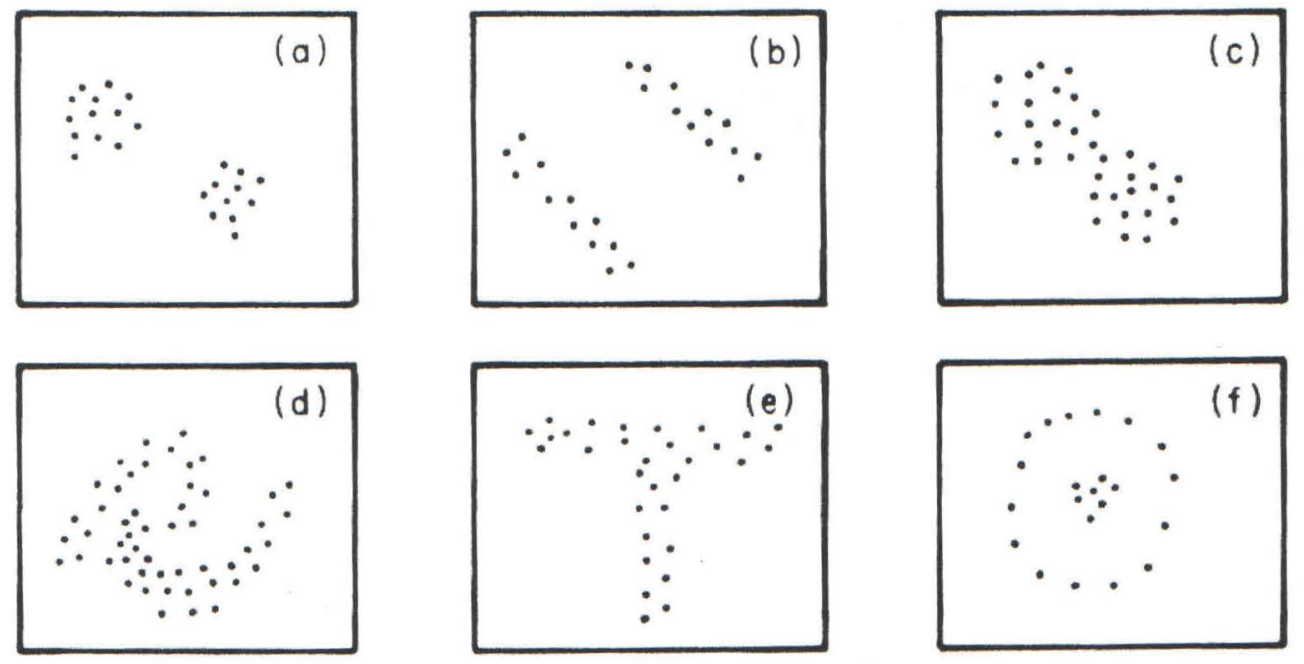

Figura 9

querendo ilustrar aqui é que os clusters podem ser bastante sensíveis à escolha particular de variáveis. Uma escolha diferente de variáveis, aparentemente e igualmente razoável, pode gerar clusters completamente diferentes. (Banchoff, 1990)

\section{Comparação de procedimentos}

Com tantos métodos disponíveis, é difícil para o pesquisador discernir o mais apropriado para um determinado grupo de condições. Vários fatores precisam ser considerados, inclusive a pergunta teórica em discussão.

Algumas condições desejáveis, para serem utilizados processos hierárquicos, segundo Willian Cleve (1993), são: considerações matemáticas, recursos computacionais, tipos de dados, tipos de variáveis envolvidas e finalidade da pesquisa. Uma 
pequena reflexão mostrará que não é fácil escrever um grupo de modelos matemáticos rígidos consensuais. A condição que sugere que os resultados produzidos por um método não deveriam depender do modo como os indivíduos são qualificados parece óbvia, porém é mais complexa do que pode aparentar. Por exemplo, o pesquisador poderia exigir do procedimento hierárquico que ele seja bem definido, isto é, que se processe de forma que possamos ter sempre a mesma árvore hierárquica no mesmo grupo de dessemelhanças observadas. A dificuldade desta condição surge, quando há dessemelhanças iguais que estão resolvidas em uma ordem arbitrária durante o processo seqüente para obtenção da árvore hierárquica.

Talvez a condição mais crucial seja a condição de continuidade sugerida por Thomas Erickson em 1993. "Pequenas" mudanças nos dados só devem produzir "pequenas" mudanças na árvore resultante. Mudanças "pequenas" nas dessemelhanças podem acontecer, se as observações estão sujeitas a erros, entretanto o pesquisador deve tomar o cuidado de distinguir entre mudanças em dessemelhanças e mudanças na matriz original de dados (n x p). Com alguns métodos de obtenção de cluster, mudanças "pequenas" nos dados podem produzir "grandes" mudanças na árvore resultante, e isto é certamente indesejável. Um exemplo interessante é determinado por Erickson (1993), onde o método hierárquico single-link é usado para produzir mudanças contínuas na estrutura de árvore, enquanto o método hierárquico complete-link e group-average geram mudanças descontínuas na estrutura da árvore hierárquica.

Outro grupo importante de condições é citado por Erickson (1993). São as chamadas condições de "ajuste-mútuo". Em particular, se o pesquisador soma ou subtrai um indivíduo do grupo original, o esperado é uma mudança relativamente pequena na estrutura da árvore, entretanto, às vezes, a classificação será mudada de modo não trivial. Uma possível exigência é que o pesquisador elimine todos os indivíduos de um ramo da árvore, então a estrutura restante deveria ser invariante, quando o método fosse reaplicado ao grupo restante de indivíduos.
Por que alguns métodos trabalham bem com certos tipos de dados e não com outros? Os estudos empíricos enfatizam o fato de que a maioria dos métodos fazem suposições implícitas sobre a estrutura em uma amostra. Se estas suposições não são suficientes, ou são falsas, um método hierárquico pode impor uma estrutura não verdadeira nos dados, de forma que soluções espúrias possam ser adotadas. Por exemplo, alguns métodos tendem a achar estruturas esféricas para clusters, qualquer que seja a verdadeira forma (se acontecer dos dados terem realmente a estrutura esférica, este obviamente será o melhor método). Como um segundo exemplo, notamos que, se uma técnica é utilizada para dividir os dados em cinco clusters, então ela o fará, mesmo que o número natural de clusters seja menor ou maior que cinco (caso este número esteja muito longe de cinco, diferentes técnicas matemáticas forçarão a um erro maior ou menor). Como um terceiro exemplo, a técnica que minimiza o determinante ou a matriz do método hierárquico within-group pela soma dos quadrados e produtos, efetivamente assume que todos os grupos têm a matriz de covariância zero. Quando este não é o caso, o método pode produzir respostas completamente equivocadas. Como um quarto exemplo, foi pedido a pesquisadores para tecerem comentário sobre os resultados do método hierárquico de Wishart em alguns dados adotando como parâmetro $\mathrm{k}=4$. Os especialistas foram surpreendidos, pois um cluster conhecido (de dois indivíduos) não foi encontrado pelo método. A razão é simples. Escolhendo o parâmetro $\mathrm{k}=4$, estamos dizendo efetivamente que todos os grupos têm que conter quatro indivíduos pelo menos. (Dean 1992)

Tendo em mente a ampla variedade de situações práticas, é bastante difícil fazer recomendações gerais. A sugestão para o analista é tentar vários métodos diferentes de obtenção de cluster e, se alguns deles revelam os mesmos clusters, então a pessoa pode estar confiante que clusters naturais realmente existem. Mas os estudos empíricos demonstram que isto é improvável de acontecer, a menos que os grupos sejam amoldados de forma esférica e bem separados. 


\section{Conclusão}

Devemos fechar este assunto com algumas notas precautórias e diretrizes recomendadas ao uso apropriado das metodologias de agrupamento:

a) a maioria das técnicas de agrupamento são sensíveis à presença de outhers, devendo esses serem removidos antes da análise;

b) técnicas hierárquicas devem ser usadas preferencialmente em casos onde os dados possuem grupos hierárquicos naturais. Por exemplo, no estudo da zoologia;

c) a pergunta: "qual deve ser a medida de distância similar", ainda está sem resposta;

d) estudos em campos de pesquisas temporais parecem favorecer o uso do método de lincagem comum, pois tal método facilita a formatação de agrupamentos baseados em medidas de critérios internos; e) técnicas de otimização, enquanto oferecem grandes perspectivas, dificilmente são usadas em grandes quantidades de dados, por motivos operacionais;

f) mininizando IWI há vantagens em cima da minimização de $\operatorname{tr}(W)$. Por exemplo, minimizando IWI, resultados excelentes foram obtidos em aplicações empíricas pesquisadas por Friedman e Rubin (1967); este método não tem nenhuma restrição sobre a forma dos agrupamentos e pode ser facilmente operacionalizado;

g) recomendamos que, sempre que possível, os dados sejam divididos e cruzados, para avaliar a estabilidade de soluções de agrupamento.

A análise de cluster freqüentemente é o primeiro passo em uma análise de dados. Por conseguinte, ela deve conduzir naturalmente a outras pesquisas adicionais, e não simplesmente induzir o pesquisador à aceitação casual dos agrupamentos obtidos.

\section{REFERÊNCIAS BIBLIOGRÁFICAS}

ABRAM,G.; TREINISH, L. An Extended Data-Flow Architecture for Data Analysis and Visualization. New York: Computer Graphics, 1999.

AHLBERG C., C. WILLIAMSON; B. SHNEIDERMAN. Dynamic Queries for Information Exploration: An Implementation And Evaluation. New York: Chapman and Hall, 1997.

ANGEHRN, A. A. Modeling by Example: A Link Between Users, Models and Methods Journal of Operational Research, Minnesota, $55: 296-308,1992$.

ARNHEIM, Rudolf. Arte e Percepção Visual. 10. ed. São Paulo: Pioneira, 1996.

BANCHOFF, T. F. Visualizing Two-Dimensional Phenomena in Four-Dimensional Space: A Computer Graphics Approach. New York: J. Wegman and D. J. DePriest, Editors, 1986.

BANCHOFF, T. F. Beyond the Third Dimension: Geometry, Computer Graphics and Higher Dimensions. New York: Freeman, 1990. CHATFIELD, Cristopher. V. COLLINS, Alexander. Introduction to Multivariate Analysis. New York: Chapman e Hall, 1992.

CHATFIELD, Cristopher. Statistic and Computer. New York: Chapman e Hall, 1986.

CORMACK, R.M. A Review of Classification. New York: J.R. Biometryka, 1971.

EVARITT, Brian. Cluster Analysis. 2. ed. New York: Halsted Press,1980.

KRZANOWSKI, W. V. Principles of Multivariate Analysis. Oxford: Clarendon Press, 1993.

MACKINLAY, J. D.; ROBERTSON, G. G.; CARD, S. K. The Perspective Wall: Detail and Context Smoothly Integrated. New York: Robertson Editors, Interaction Conference on Human Factors in Computing Systems, 173-179, 1991.

ROBERTSON, G. G.; MACKINLAY, J. D.; CARD, S. K. Visualizations of Hierarchical Information. New York: Robertson Editors. Interaction Conference on Human Factors in Computing Systems, 189-194, 1991.

SPIGEL, J.; GOLGWYN, R. ; FRIEDMAN, H. Outliers in Statistical Data. New York: Jonh Wiley, 1971.

WANG, Peter C. C. Graphical Representation of Multivariate Data. London: Academia Press, 1978. 